\title{
Environnement familial et bien-être économique des enfants américains
}

\section{THE LIVING ARRANGEMENTS AND ECONOMIC WELL-BEING OF AMERICAN CHILDREN MEDIO FAMILIAR Y BIENESTAR ECONÒMICO DE LOS NINOS AMERICANOS}

\section{Daniel T. Lichter}

Volume 23, numéro 2, automne 1994

L'enfance (suite)

URI : https://id.erudit.org/iderudit/010169ar

DOI : https://doi.org/10.7202/010169ar

Aller au sommaire du numéro

Éditeur(s)

Association des démographes du Québec

ISSN

0380-1721 (imprimé)

1705-1495 (numérique)

Découvrir la revue

Citer cet article

Lichter, D. T. (1994). Environnement familial et bien-être économique des enfants américains. Cahiers québécois de démographie, 23(2), 151-177. https://doi.org/10.7202/010169ar
Résumé de l'article

À partir de nouvelles données américaines tirées de l'échantillon à $1 \%$ du Public Use Microdata Sample du Recensement décennal de 1990, l'auteur cherche à mesurer à quel point les variations interraciales du bien-être économique chez les enfants sont liées à des différences entre les groupes de diverses ethnies ou races au point de vue de la composition familiale et (ou) de la participation des parents au travail rémunéré. Il apparaît que les différences interraciales de composition familiale rendent inopérants les efforts entrepris pour éliminer les écarts de richesse entre enfants américains de diverses origines. Ainsi, l'importance de la proportion d'enfants noirs vivant en famille monoparentale avec leur mère explique $60 \%$ de l'écart qui sépare le taux de pauvreté des enfants noirs de celui des enfants blancs. De même, les écarts de pauvreté chez les enfants entre les groupes s'expliquent en partie (mais non complètement) par la variation interraciale des modèles de participation au travail des parents. Parmi les enfants vivant en famille biparentale, les taux de pauvreté sont environ deux fois plus élevés chez les enfants noirs que chez les enfants blancs, même si les premiers ont plus de chances d'avoir deux parents qui travaillent. On peut en conclure que les inégalités raciales ne seront pas éliminées par les politiques strictement conçues pour «renforcer la famille» ou inciter les mères au travail (sans égard au niveau des salaires), surtout dans le cas des enfants des minorités défavorisées depuis plusieurs générations.
Tous droits réservés @ Association des démographes du Québec, 1994

Ce document est protégé par la loi sur le droit d'auteur. L'utilisation des services d'Érudit (y compris la reproduction) est assujettie à sa politique d'utilisation que vous pouvez consulter en ligne.

https://apropos.erudit.org/fr/usagers/politique-dutilisation/ 
Cahiers québécois de démographie

Vol. 23, no 2, automne 1994, p. 151-177.

\title{
Environnement familial et bien-être économique des enfants américains
}

\author{
Daniel T. LICHTER *
}

Les enfants américains ont aujourd'hui presque deux fois plus de chances d'être pauvres que les personnes âgées. Leur taux de pauvreté, qui a grimpé d'environ le tiers depuis quinze ans, dépasse de loin celui des enfants des autres pays industrialisés (Galston, 1993; Corbett, 1993; Bianchi, 1993; National Commission on Children, 1991). Il se situait en effet à 21,9\% en 1992 (U. S. Bureau of the Census, 1993), soit le niveau le plus élevé jamais atteint pour une année depuis la récession de 1983. Le nombre actuel d'enfants pauvres - 14,6 millions - dépasse tous les totaux annuels enregistrés aux États-Unis depuis 1965 (U. S. Bureau of the Census, 1993). De plus, les écarts de revenu entre enfants riches et enfants pauvres se sont considérablement accentués au cours de la décennie 1980, et le revenu réel des seconds a subi un déclin absolu (Lichter et Eggebeen, 1993; Hernandez, 1993).

La détérioration de la situation économique des enfants est un fait communément admis (Easterlin, 1987; Danzinger et

* Population Research Institute, Pennsylvania State University. Cet article contient des extraits de "Children and Youth: Living Arrangements and Welfaren, à paraittre dans Reynolds Farley, éd., State of the Union: Americans in the 1990s, vol. 2, Social Trends, () Russell Sage Foundation, 1995. Nous avons bénéficié pour cette recherche de subventions octroyées par la National Science Foundation (SES-8921249) (volet "Poverty, Spatial Inequality and the Changing Living Arrangements and Welfare of Children and Youth [Pauvreté, inégalités spatiales et évolution de l'environnement familial et du bien-être des enfants et des jeunes]") et par la Russell Sage Foundation (volet "The Living Arrangements and Welfare of Children and Youth [L'environnement familial et le bien-être des enfants et des jeunes]"). Le Population Research Institute jouit du soutien du National Institute of Child Health and Human Development (P30 HD28263-01). Traduction : Johanne Archambault, sous la supervision de Nicole Marcil-Gratton et de Céline Le Bourdais. 
Danzinger, 1993), mais on s'entend beaucoup moins sur les facteurs de la pauvreté et de l'insuffisance de revenus, surtout en ce qui concerne les enfants des minorités raciales et ethniques (Bianchi et McArthur, 1991; Lichter et Landale, 1995). Nous nous proposons, dans cet article, de mesurer à quel point les diffêrences de bien-être économique observées entre les enfants de divers groupes raciaux ou ethniques sont liées à des différences entre ces communautés au point de vue des structures familiales - ou, plus exactement, de la composition de l'entité parentale : couple marié, femme seule, homme seul, etc. - et des modes de participation du ou des parents au travail rémunéré. Cette étude sera la première à exploiter les données sur les enfants du fichier à $1 \%$ du Public Use Microdata Sample ${ }^{1}$ (PUMS) du Recensement décennal de 1990, fichier disponible depuis peu. Elle nous permettra de faire ressortir les principaux effets qu'entraînent, pour le bien-être économique des enfants, la non-participation et la participation partielle du ou des parents à la main-d'œuvre.

\section{LA PAUVRETÉ CHEZ LES ENFANTS AMÉRICAINS : QUELgUES FAITS}

Les conséquences sociales et économiques de la pauvreté chez les enfants, en particulier de la pauvreté prolongée ou chronique, sautent aux yeux et sont souvent inquiétantes. Par rapport aux autres enfants, les enfants pauvres risquent davantage de souffrir de mauvaise santé, de réussite scolaire inférieure à la moyenne (c'est-à-dire de taux de décrochage supérieurs) et de pauvreté à l'âge adulte (voir par exemple McLanahan, 1985; Children's Defense Fund, 1991). La situation économique actuelle des enfants américains laisse présager un avenir plein d'incertitudes pour les générations d'adultes qu'ils formeront. Les problèmes liês à la pauvreté vécue durant l'enfance (tels les comportements de décrochage scolaire et la grossesse prémaritale) peuvent en effet nuire à la compétitivité de la main-d'œuvre américaine au sein de l'économie mondiale. Aussi se demande-t-on de plus en plus si le pays, par ses politiques, investit suffisamment dans ses enfants (Haveman et Wolfe, 1993; Fuchs et Reklis, 1992; Macunovich et Easterlin, 1990).

1 Échantillon de microdonnées à grande diffusion (NDLT). 
À ce jour, la plupart des études ont porté surtout sur les conséquences économiques de la transformation des cadres familiaux (Hernandez, 1993; Bianchi et McArthur, 1991). On ne s'en étonne pas quand on sait que l'augmentation du nombre de familles monoparentales à chef fêminin expose un pourcentage croissant d'enfants américains à la pauvreté et au dénuement (Duncan et Rodgers, 1991; Eggebeen et Lichter, 1991). À peu près la moitié de l'augmentation de la pauvreté chez les enfants enregistrée après 1980 correspond à l'évolution de la proportion d'enfants vivant dans une famille monoparentale "à risque élevén, en particulier dans une famille pauvre dirigée par une femme (Eggebeen et Lichter, 1991). Le taux de pauvreté des familles à chef féminin avec enfants, remarquablement stable depuis 25 ans, atteignait $45,7 \%$ en 1992 (U. S. Bureau of the Census, 1993). Chez les noirs, il s'élevait à 57,2\%.

Il semble clair désormais que dans la société américaine la composition familiale est de plus en plus étroitement liée à l'inégalité raciale (Eggebeen et Lichter, 1991; Hernandez, 1993). Environ deux tiers des enfants afro-américains naissent hors des liens du mariage, et seulement une minorité d'enfants noirs vit actuellement en famille biparentale. La situation des enfants portoricains est comparable (Landale et Huaun, 1993). L'accroissement manifeste des différences interraciales de composition familiale (Cherlin, 1981) laisse supposer que les inégalités raciales - économiques ou autres - sont accentuées par les transformations familiales actuelles. Eggebeen et Lichter (1991) ont montré qu'en 1987 environ $60 \%$ des écarts de pauvreté entre blancs et noirs étaient liés aux différences interraciales de composition familiale. Or, en 1960, la part de ces écarts associée à des différences interraciales eu égard aux proportions d'enfants vivant dans une famille dirigée par un couple marié et d'enfants vivant en famille monoparentale était à peu près nulle. Hernandez (1993) aboutit à des conclusions similaires. Si l'augmentation du nombre de familles à chef féminin postérieure à 1960 ne s'était pas produite, les écarts de pauvreté chez les enfants entre blancs et noirs n'auraient atteint que la moitié ou les trois quarts du niveau observé. Malheureusement, les inégalités raciales risquent fort de s'aggraver lorsque la présente cohorte d'enfants noirs défavorisés atteindra l'âge adulte et que les conséquences à long terme de la pauvreté des enfants se seront pleinement manifestées. 
Tandis que les transformations familiales semblaient vouer à la pauvreté des proportions d'enfants de plus en plus fortes, les mesures sociales visant à favoriser l'insertion sur le marché du travail des parents et en particulier des mères célibataires et des mères pauvres se sont progressivement imposées dans l'opinion comme la meilleure solution au problème de la pauvreté (Haveman et Wolfe, 1993; Mead, 1993). Ainsi vient-on de réaffirmer, à la faveur du débat sur la pauvreté, l'importance de l'autonomie économique et de la famille biparentale comme cadre idéal pour l'éducation des enfants. Dans cet esprit, les nouvelles modifications apportees aux programmes sociaux (par exemple les mesures favorables aux couples mariés ${ }^{2}$ ) font la promotion de la famille biparentale, comme si elle pouvait être la panacée qui résoudra le problème de la pauvreté. De même, le Job Opportunities and Basic Skills Training Program (JOBS) créé par le Family Support Act ${ }^{3}$ de 1988 a accru le rôle premier de l'autonomie économique (c'est-à-dire du travail) comme stratégie de lutte contre la pauvreté des familles et des enfants.

Ces initiatives témoignent d'une réalité devenue incontournable : de plus en plus, les enfants pauvres vivent de prestations sociales, tandis que l'apport du revenu de travail des parents diminue (Jensen, Eggebeen et Lichter, 1993). En 1969, selon Hernandez (1993), 61,8\% des enfants pauvres étaient totalement autosuffisants, au sens qu'ils ne recevaient pas d'aide sociale. En 1988, seulement 44,0 \% étaient dans ce cas. Chez les enfants noirs, la proportion est passée de $51,0 \%$ à $29,1 \%$ durant la même période. On doit en conclure que les différences interraciales touchant les modes de participation au marché du travail des parents des enfants pauvres ont contribué aux différences interraciales relatives à la situation de pauvreté des enfants, en particulier aux différences entre blancs et noirs. Malheureusement, et l'on peut s'en étonner, rares sont les travaux empiriques consacrés à la relation entre le travail des parents ou la composition familiale et les différences interraciales de bien-être économique chez les enfants.

2 "Wedfare" programs. Contraction (intraduisible) de welfare (assistance sociale) et de wed (se marier); à comparer à workfare ("work for welfare") (NDLT).

3 Programme d'accessibilité à l'emploi et d'acquisition des compétences de base, prévu par la loi sur le soutien à la famille; JOBS, sigle correspondant au nom du programme, signifie "emplois" (NDLT). 
Les éléments que nous apportons au débat sont les suivants. Tout d'abord, notre examen porte sur des groupes ethniques ou raciaux (enfants asiatiques, latino-américains et amérindiens notamment) que les analyses antérieures avaient dû laisser de côté, car le Current Population Survey et autres enquêtes à passages répétés, tel le Panel Survey of Income Dynamics ${ }^{4}$, ne fournissent pas d'échantillons de taille suffisante dans leur cas (Eggebeen et Lichter, 1991; Lichter et Eggebeen, 1994; Duncan, 1991). Les travaux existants portent presque exclusivement sur l'inégalité entre blancs et noirs.

En second lieu, nous essaierons de mesurer jusqu'à quel point les différences interraciales de pauvreté chez les enfants sont liées à des différences entre les divers groupes au point de vue des modes d'insertion des parents sur le marché du travail : non-participation, travail à temps partiel et travail à temps plein par exemple. Jusqu'ici, les chercheurs se sont surtout intéressés au prestige professionnel, sans égard au caractère plus ou moins épisodique du travail rémunéré ou à la quantité d'heures qui lui est consacrée. Nous cherchons ainsi à mettre en lumière le rôle que pourraient jouer les différences entre les groupes eu égard au régime de travail des parents pour expliquer l'importance et la persistance des différences interraciales de pauvreté et de revenu chez les enfants.

Troisièmement, contrairement aux recherches antérieures, celle-ci tient compte des effets conjugués de la composition familiale et du régime de travail des parents sur les taux de pauvreté des enfants, pour divers groupes raciaux. Le plus souvent, on évalue les effets de la composition familiale, par exemple, sans tenir compte du travail des parents (Duncan et Rodgers, 1991; Eggebeen et Lichter, 1991; Easterlin, 1987). Les inégalités raciales chez les enfants seraient-elles moindres si les minorités raciales et ethniques présentaient les mêmes caractéristiques que la majorité blanche au chapitre de la composition familiale et du mode d'insertion des parents sur le marché du travail ?

\section{DONNÉES ET MÉTHODES}

Notre analyse repose, comme nous l'avons signalé, sur l'échantillon à $1 \%$ du Public Use Microdata Sample (PUMS), et elle se limite aux enfants de 0 an à 17 ans. Chacun fait l'objet

4 Dynamique du revenu. NDLT. 
d'un dossier indiquant si, au moment de l'enquête, il vivait dans un ménage privé ou en milieu institutionnel. L'échantillon comprend quelque 653000 individus dont la plupart $(69,1 \%)$ sont des blancs non latino-américains. Les enfants afro-américains constituent $14,6 \%$ de l'ensemble. Les autres enfants sont asiatiques $(2,9 \%)$, amérindiens $(0,9 \%)$, latinoaméricains $(12,1 \%)$ ou d'une autre ethnie "non blanche" $(0,4 \%)$.

Nous nous servirons, pour une grande partie de notre analyse, de la mesure conventionnelle (officielle) de la pauvreté basée sur les seuils définis en 1964 par Mollie Orshansky pour l'Administration de la sécurité sociale. Une famille est dite pauvre si son revenu monétaire familial de toutes sources est inférieur au seuil de faible revenu établi en fonction du prix du panier d'épicerie "économique" composé pour une famille de sa taille. Notons que cette définition ne permet pas d'additionner le revenu de deux conjoints vivant en union libre. Nous complétons cette mesure de la pauvreté par les ratios médians "revenu sur pauvreté". Le ratio revenu sur pauvreté permet de voir de combien les enfants sont au-dessus ou au-dessous du seuil de pauvreté. C'est essentiellement une mesure du revenu familial des enfants graduée selon la taille de la famille. Il synthétise et concrétise l'écart des divers groupes par rapport au seuil de pauvreté.

La méthode d'analyse que nous employons est la "standardisation directe" (Murdock et Ellis, 1991), qui consiste à se demander, en l'occurrence, quel serait le taux de pauvreté des enfants afro-américains s'ils présentaient le même profil que les enfants blancs pour la composition familiale ou le mode d'insertion des parents sur le marché du travail. L'écart entre taux observés et taux normalisés représente le rôle des différences interraciales de modèles familiaux et de modèles de travail dans l'explication des différences interraciales de pauvreté chez les enfants.

\section{RÉSULTATS}

\section{Le milieu de vie familiale des enfants}

Avec qui les enfants américains vivent-ils ? La figure 1 présente les pourcentages respectifs de ceux qui, en 1990, habitaient avec deux parents, un parent, d'autres membres de leur parenté ou d'autres personnes non apparentées, vivaient 


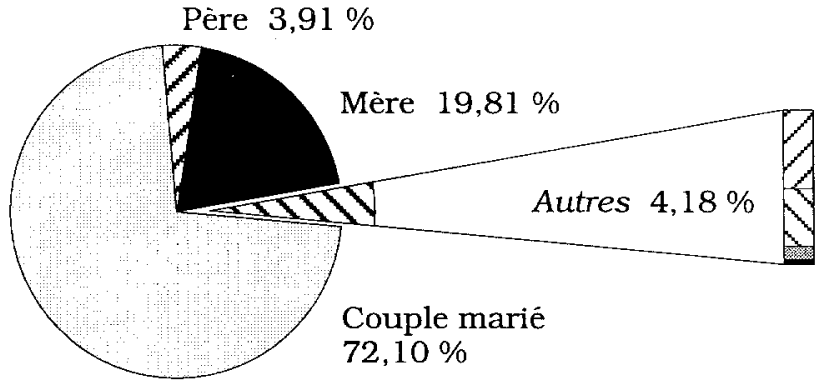

FIGURE 1 - Distribution des enfants de 0 an à 17 ans selon la composition familiale, États-Unis, 1990
Autres parents $50,96 \%$

Non-parents $37,32 \%$

En groupe $8,85 \%$

Chef ou conjoint duchef

$2,87 \%$

"en groupe", ou étaient eux-mêmes chefs de ménage ou conjoints du chef. En cette période de transformations familiales accélérées, on oublie peut-être trop facilement que la plupart des enfants américains (plus de $70 \%$ en fait) continuent de vivre en famille biparentale. De plus, environ $20 \%$ des enfants vivent en famille monoparentale avec leur mère. Pour le reste, bien qu'il ait beaucoup progressé (relativement) depuis une dizaine d'années, le pourcentage d'enfants vivant avec leur père n'atteint pas $4 \%$, et les quatre autres modes d'organisation familiale présentent des pourcentages si faibles que même réunis ils dépassent à peine ce chiffre. S'il est vrai que les enfants risquent de plus en plus de voir divorcer leurs parents (Bumpass, 1984) et que la proportion des naissances hors-mariage s'accroit, une écrasante majorité d'enfants vit donc encore, à quelque moment qu'on les observe, dans une famille dirigée par un couple marié. Évidemment, ce couple peut comprendre les deux parents biologiques, un ou des parents adoptifs, un beau-parent...

On observe néanmoins de fortes variations selon la race et l'ethnie. Alors que plus de $80 \%$ des enfants blancs non latinoaméricains vivent dans une famille biparentale, près de $50 \%$ des enfants afro-américains vivent en famille monoparentale avec leur mère (tableau 1). Ces chiffres sont beaucoup plus élevés que ceux que l'on obtient pour d'autres groupes depuis longtemps considérés comme défavorisés. Ainsi, $30 \%$ des enfants amérindiens vivent avec leur mère et $56 \%$ en famille biparentale, et près des deux tiers des enfants latinoaméricains vivent dans une famille dirigée par un couple marié. Comme la composition familiale est inséparable du bien-être économique, les différences de structure familiale entre les divers groupes risquent de rendre inopérants les 
TABLEAU 1 - Distribution des enfants de $O$ an à 17 ans de diverses origines raciales et ethniques, selon la composition familiale, EtatsUnis, 1990 (\%)

Blancs Noirs Latino- Asia- Amérin-
amér. tiques diens

L'enfant vit :

Avec ses deux parents Avec son père Avec sa mère Avec d'autres parents Avec des non-parents En groupe L'enfant est le chef de famille ou son conjoint TOTAL

$\begin{array}{rrrrr}80,6 & 37,4 & 64,0 & 84,2 & 56,3 \\ 3,2 & 5,5 & 5,9 & 2,8 & 6,7 \\ 13,3 & 48,8 & 24,0 & 9,4 & 30,1 \\ 1,2 & 5,5 & 3,2 & 2,1 & 3,8 \\ 1,4 & 1,9 & 2,3 & 1,3 & 2,3 \\ 0,3 & 0,8 & 0,5 & 0,2 & 0,7\end{array}$

$\begin{array}{lllll}0,1 & 0,1 & 0,2 & 0,1 & 0,2\end{array}$ $100,0 \quad 100,0 \quad 100,0 \quad 100,0 \quad 100,0$

efforts entrepris pour promouvoir l'égalité économique interraciale (Bianchi et McArthur, 1991; Hernandez, 1993).

Les données du recensement décennal de 1990 montrent que le cadre familial des enfants change notablement d'un groupe racial ou ethnique à l'autre au sein de la société américaine. Comme nous le verrons, il en va de même pour la situation économique, ce qui laisse croire qu'il existe un lien entre la composition familiale et le manque de ressources. Il reste à savoir si c'est la pauvreté qui met en péril les modèles familiaux traditionnels (la famille dirigée par un couple marié) ou la précarité du mariage et la fécondité extra-maritale qui entraînent ou aggravent les difficultés matérielles de certaines minorités raciales et ethniques. À défaut de vider cette question, nous allons présenter des données susceptibles de l'éclairer.

\section{Pauvreté et dénuement chez les enfants américains}

Il ne fait aucun doute que le visage de la pauvreté rajeunit aux États-Unis (Preston, 1984). En 1969, le taux de pauvreté des personnes âgées atteignait $24,6 \%$, pourcentage très supérieur au taux de pauvreté global. En 1989, il n'était plus que de $11,4 \%$. Mais parallèlement, le taux de pauvreté des moins de 18 ans était passé de $14,0 \%$ à $19,6 \%$.

Or, comme on le voit au tableau 2 , si le taux de pauvreté global des enfants vivant avec leurs parents ou avec d'autres personnes de leur parenté se situe à $17,6 \%$, il existe de fortes 
TABLEAU 2 - Taux de pauvreté (\%) et ratios médians revenu/pauvreté, enfants de $0-17$ ans, par groupe racial ou ethnique, $1990^{\mathrm{a}}$

\begin{tabular}{lcc}
\hline $\begin{array}{l}\text { Enfants de } \\
\text { G-17 ans }\end{array}$ & $\begin{array}{c}\text { Taux de } \\
\text { pauvreté }\end{array}$ & $\begin{array}{c}\text { Ratio médian } \\
\text { revenu/pauvreté }\end{array}$ \\
\hline Total & 17,6 & 247 \\
Blancs & 10,5 & 280 \\
Noirs & 38,9 & 136 \\
Latino-américains & 31,3 & 156 \\
Asiatiques & 16,8 & 290 \\
Amérindiens & 38,6 & 131 \\
\hline
\end{tabular}

a. Enfants vivant avec leurs parents ou leur parentèle seulement.

variations autour de cette moyenne. Le taux de pauvreté des enfants blancs est d'un peu plus de $10 \%$, par exemple, tandis que celui des enfants afro-américains et des enfants amérindiens avoisine $39 \%$. Près du tiers des enfants latino-américains sont pauvres mais, au sein de ce groupe, les enfants cubains ont un taux de $14,4 \%$ (le plus bas) tandis que les enfants portoricains ont un taux de plus de $40 \%$; et près du tiers des enfants mexicano-américains sont pauvres selon la définition gouvernementale officielle (données non présentées). Ces disparités donnent raison à ceux qui veulent que l'on cesse d'analyser les Latino-américains comme un groupe homogène.

Le tableau 2 présente aussi, pour chaque groupe racial ou ethnique, le ratio médian "revenu sur pauvreté" (multiplié par 100). Le revenu familial médian des enfants américains est presque deux fois et demie supérieur au seuil de pauvreté (ratio de 247). Les enfants blancs non latino-américains et les enfants asiatiques se situent encore plus haut (280 et 290), tandis que les enfants afro-américains et amérindiens ne dépassent que légèrement le seuil (ratios de 136 et 131 respectivement).

Comme nous nous y attendions, le taux de pauvreté et le ratio revenu sur pauvreté sont très sensibles à la composition familiale (tableau 3). Par rapport à l'ensemble, les enfants vivant en famille biparentale ont, de loin, le taux de pauvreté le plus faible $(8,9 \%)$ et le ratio revenu sur pauvreté le plus élevé (285). Les enfants qui vivent avec leur mère ont un taux de pauvreté de $46 \%$ et un revenu proche de la pauvreté (ratio de 112). Il y a nettement plus de pauvreté chez ces enfants que chez 
TABLEAU 3 - Taux de pauvreté (\%) et ratios médians revenu/ pauvreté, enfants de $0-17$ ans par groupe racial ou ethnique, selon la composition familiale, 1990

$\begin{aligned} & \text { Composition Groupes Total Blancs Noirs Latino- Asia- Amérin- } \\ & \text { familiale }\end{aligned}$
amér. tiques diens

L'enfant vit avec:

TAUX DE PAUVRETÉ

Ses deux parents

Son père

Sa mère

D'autres parents

$\begin{array}{rrrrrr}8,9 & 6,2 & 14,7 & 21,8 & 13,7 & 25,1 \\ 22,9 & 15,1 & 33,9 & 33,0 & 19,8 & 49,1 \\ 46,1 & 34,4 & 57,1 & 55,4 & 39,0 & 60,4 \\ 34,1 & 20,5 & 46,8 & 35,7 & 34,5 & 45,9\end{array}$

L'enfant vit avec:

RATIOS MÉDIANS REVENU/PAUVRETÉ

\begin{tabular}{lrrrrrr} 
Ses deux parents & 285 & 303 & 231 & 185 & 311 & 178 \\
Son père & 199 & 238 & 145 & 141 & 237 & 110 \\
Sa mère & 112 & 152 & 79 & 85 & 153 & 77 \\
D'autres parents & 153 & 204 & 104 & 138 & 186 & 108 \\
\hline
\end{tabular}

ceux qui vivent avec leur père ou avec d'autres parents. Quant à savoir si les enfants qui vivent avec leur père jouissent d'une très bonne situation économique (si le père en a la garde parce qu'il est à l'aise financièrement), on note que ces enfants ont des taux de pauvreté supérieurs à ceux de l'ensemble.

Ces données soulèvent l'hypothèse d'un lien entre les différences interraciales de composition familiale et les diffèrences interraciales de pauvreté et de bien-être économique chez les enfants. Quelle est la force de ce lien ? Telle est la question que nous abordons maintenant.

\section{Composition familiale et pauvreté}

La composition familiale est indissociable de la sécurité économique des enfants. C'est le constat qui se dégage du tableau 3, où apparaissent les taux de pauvreté par groupe racial ou ethnique selon la composition familiale. Dans tous les groupes, les taux de pauvreté les plus élevés et les ratios revenu sur pauvreté les plus faibles sont ceux des enfants qui vivent avec leur mère; le taux de pauvreté des enfants vivant sans leur père est exceptionnellement élevé chez les enfants afro-américains et les enfants amérindiens (environ $60 \%$ ). Ces niveaux sont d'autant plus alarmants qu'ils se maintiennent à un moment où la proportion d'enfants américains vivant en famille monoparentale avec leur mère va croissant. 
Il n'empêche que les taux de pauvreté atteignent leur maximum chez les enfants des minoritês, peu importe la composition familiale. Il est donc clair que, d'un groupe à l'autre, les différences de forme familiale ne sont pas la seule explication des différences de bien-être économique constatées chez les enfants. Aussi la création de familles biparentales n'éliminera-t-elle pas le problème de la pauvreté chez les enfants des minorités. Le taux de pauvreté des enfants qui vivent avec leur père et leur mère, à son niveau le plus bas chez les blancs non latino-américains (6\%), s'élève à $22 \%$ chez les enfants latinoaméricains et à $25 \%$ chez les enfants amérindiens. Ce sont là des écarts considérables à tous égards.

Les variations du bien-être économique associées à la composition familiale sont particulièrement marquées au sein de certains groupes. Chez les blancs, par exemple, les enfants qui vivent avec leur mère ont un taux de pauvreté 5,5 fois plus élevé que les enfants qui vivent avec leurs deux parents. Dans le groupe afro-américain, les enfants vivant avec leur mère sont 3,9 fois plus susceptibles d'être pauvres que les enfants qui vivent avec leur père et leur mère.

Si ces résultats attestent l'existence d'un lien étroit entre la composition familiale et les situations de pauvreté chez les enfants, il saute aux yeux que les taux de pauvreté élevés des groupes dits défavorisés ne mettent pas seulement la famille en cause. Examinons la variation du taux de pauvreté normalisé et celle du ratio "taux de pauvreté normalisé sur taux de pauvreté observé" en fonction de la composition familiale (tableau 4); le ratio exprime l'écart entre le taux de pauvreté réel des enfants d'un groupe et le taux qu'ils auraient si on les transposait dans les modèles familiaux des enfants blancs.

TABLEAU 4 - Taux de pauvreté des enfants de 0 an à 17 ans, normalisés par référence à la composition familiale des blancs, selon la race ou l'origine ethnique, 1990

\begin{tabular}{lcc}
\hline & $\begin{array}{c}\text { Taux de } \\
\text { pauvreté } \\
\text { normalisé } \\
(\%)\end{array}$ & $\begin{array}{c}\text { Taux norma- } \\
\text { lisé/Taux } \\
\text { observé }\end{array}$ \\
\hline Noirs & 21,5 & \\
Latino-américains & 26,9 & 0,55 \\
Asiatiques & 17,6 & 0,86 \\
Amérindiens & 30,9 & 1,05 \\
\hline
\end{tabular}


Le ratio de 0,55 obtenu pour les enfants afro-américains, par exemple, signifie que leur taux normalisé équivaut à seulement $55 \%$ de leur taux observé ou, inversement, que leur taux de pauvreté serait de $45 \%$ inférieur s'ils avaient les mêmes formes familiales que les enfants blancs. En 1960, les différences de modèles familiaux entre enfants blancs et enfants noirs n'expliquaient à peu près rien de leurs différences de pauvreté (Eggebeen et Lichter, 1991). Les effets de la composition familiale sont moins nets pour les groupes latinoaméricain et amérindien, si ce n'est qu'ils comptent une proportion supérieure de familles monoparentales qui y accroît légèrement le niveau de pauvreté des enfants. Il est intéressant de constater qu'au contraire, dans le groupe asiatique, à cause de la proportion de familles dirigées par un couple marié, les taux de pauvreté observés sont plus faibles que les taux qui iraient de pair avec des formes familiales identiques à celles des blancs.

Bien qu'on ne puisse faire abstraction des effets de la composition familiale, les politiques qui tendent à ériger la famille biparentale en norme ne pourront supprimer les différences interraciales de pauvreté aux États-Unis. Un autre facteur - la participation des parents au travail rémunéré semble en effet influencer les variations interraciales et interethniques de la pauvretê chez les enfants.

\section{Présence des parents sur le marché du travail et pauvreté des enfants}

Face à des transformations familiales dont bon nombre paraissent irréversibles dans l'immédiat, bien des décideurs en sont venus à considérer la participation au travail rémunéré comme le plus sûr recours des familles pauvres. De fait, les mesures d'accessibilité au travail de la loi de 1988 sur le soutien à la famille érigent le travail en panacée au problème de la pauvreté, en particulier dans le cas des familles dirigées par une mère célibataire. Il est donc opportun de prendre en considération les modes de participation des parents au marché du travail et de s'interroger sur le rôle que jouent les variations de ces modes entre les groupes dans l'explication des écarts interraciaux de pauvreté chez les enfants.

Le tableau 5 présente la distribution des enfants de chaque groupe en fonction de la composition familiale et du mode de participation du ou des parents au travail rémunéré. En 1990, 


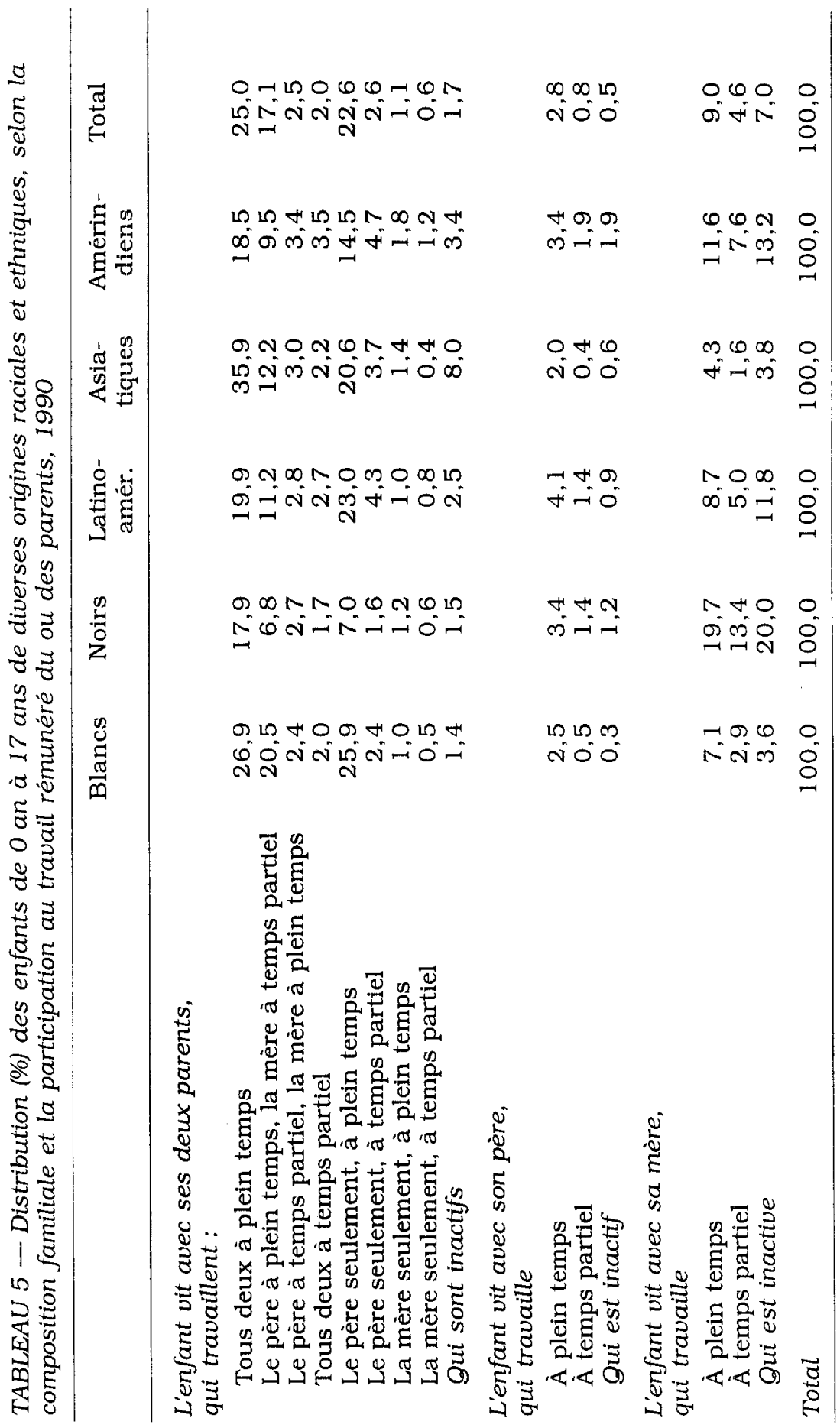


seulement $23 \%$ des enfants américains vivaient dans une famille conforme au modèle "traditionnel" : père travaillant à plein temps et mère au foyer. Aujourd'hui, la famille modale $(25 \%)$ est celle où les enfants vivent avec deux parents qui travaillent à plein temps. Le deuxième type le plus répandu (17\%) est la famille où le père travaille à plein temps et la mère à temps partiel (moins de 35 heures par semaine). De nos jours, la plupart des enfants grandissent auprès d'un ou de deux parents présents sur le marché du travail. Seulement $9 \%$ environ des enfants amêricains vivent dans une famille dirigée par un ou deux parents qui ne travaillent pas.

Le modèle traditionnel est beaucoup moins répandu dans certaines minorités. Si $26 \%$ des enfants blancs vivent avec un père qui travaille à plein temps et une mère au foyer, seulement $7 \%$ des enfants afro-américains sont dans le même cas. Le pourcentage est faible aussi chez les enfants amérindiens (15\%). C'est chez les enfants asiatiques que l'on trouve la plus forte proportion d'enfants vivant avec deux parents qui travaillent à plein temps (36\%), et chez les enfants noirs que l'on constate la plus faible (18\%) : dans leur cas, la famille modale est celle où les enfants vivent avec une mère célibataire qui reste au foyer ( $20 \%$ ).

Les pourcentages du tableau 5 sont l'expression combinée de différences relatives à la fois aux modèles familiaux et aux modes de présence des parents sur le marché du travail. Le tableau 6 met l'accent sur la distribution des enfants des divers groupes selon la participation de leurs parents au travail en distinguant entre trois formes familiales : couple marié, père célibataire et mère célibataire. Sur l'ensemble des enfants qui vivent avec un couple marié, environ le tiers ont deux parents travaillant à plein temps; près du quart ont un père travaillant à plein temps et une mère travaillant à temps partiel; et $30 \%$ ont un père travaillant à plein temps et une mère au foyer. De toute évidence, dans les familles biparentales, le travail à temps plein du père fait partie de la vie des enfants $(85 \%$ des cas) et le travail de la mère est chose courante.

Les familles monoparentales présentent une image bien différente. En ce qui concerne les enfants qui vivent avec leur père, celui-ci travaille à plein temps dans les deux tiers des cas. Et seulement $44 \%$ des mères chefs de famille monoparentale travaillent à plein temps et $34 \%$ restent au foyer. La moindre participation des parents célibataires au marché du travail s'explique en partie par la présence de jeunes enfants et par les 


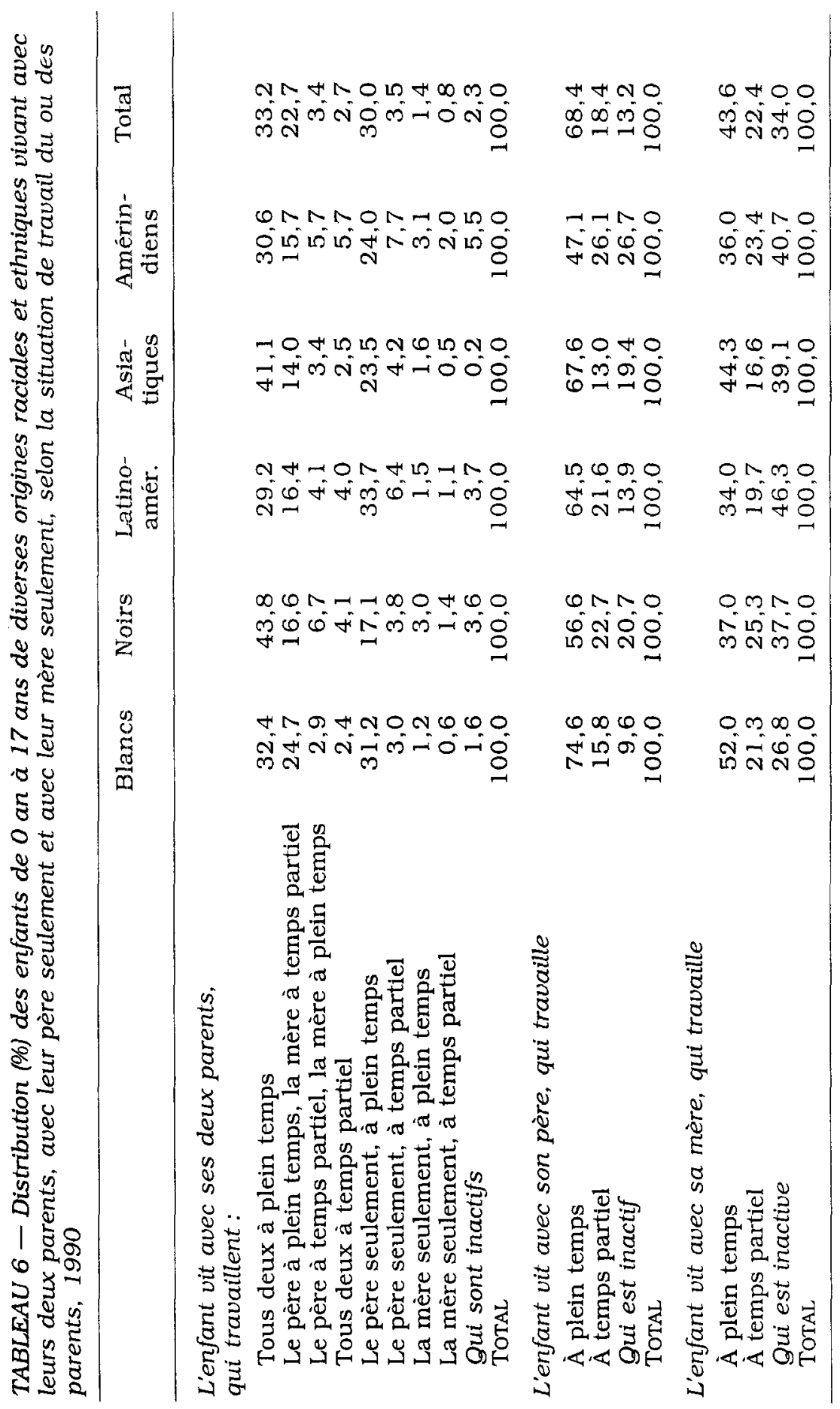


difficultés liées à leur prise en charge. C'est pourquoi l'on ne s'étonne guère que les enfants des familles monoparentales absorbent plus que leur part de la pauvreté vécue par les enfants aux États-Unis. Ils ont moins de chances d'avoir un parent présent sur le marché du travail et n'ont pas accès au surplus de richesse et de sécurité que procure le travail des deux parents. D'où, sans doute, la dépendance croissante des enfants qui vivent avec leur mère à l'égard des prestations sociales (comparativement aux revenus de travail) (Jensen, Eggebeen et Lichter, 1993).

La participation des parents au travail rémunéré est le lieu de variations interraciales qui passent trop facilement inaperçues. Sans doute notre résultat le plus frappant concerne-t-il les enfants noirs vivant en famille biparentale, qui comptent le plus haut pourcentage d'enfants dont les deux parents travaillent à plein temps (44\%), dépassant à cet égard les enfants blancs (32\%) et les enfants asiatiques (41\%). Dans ce cas-ci, les différences interraciales quant à la participation des parents à la main-d'œuvre au sein des familles biparentales ont sans doute pour effet d'atténuer la proportion de pauvres chez les enfants noirs, qui demeure néanmoins la plus forte. Le problème tient au faible pourcentage d'enfants noirs vivant en famille biparentale et, comme nous le verrons, à la faible participation à la main-d'œuvre des parents célibataires dans le cas des enfants noirs.

La participation au travail des parents des enfants noirs vivant en famille biparentale contraste de façon marquée avec celle des mères d'enfants noirs vivant en famille monoparentale. Seulement $37 \%$ de ces dernières travaillaient à temps plein, proportion beaucoup plus faible que chez les blancs (52\%). Les enfants (de mère seule) du groupe latino-américain sont les plus susceptibles d'avoir une mère inactive, mais on note une variation substantielle à l'intérieur de ce groupe. Ainsi, selon Lichter et Landale (1995), chez les enfants cubanoaméricains vivant en famille monoparentale à chef féminin, la mère travaille à plein temps dans près de $50 \%$ des cas. Les enfants portoricains présentent la proportion la plus faible: $23 \%$.

On a donc affaire à des variations raciales et ethniques considérables dans la présence des parents sur le marché du travail. Ce facteur - à l'instar des transformations familiales - semble accentuer les difficultés économiques de certains groupes raciaux et de certaines minorités perpétuellement 
défavorisés. La situation est particulièrement difficile pour les enfants noirs, qui devraient pouvoir compter plus souvent sur deux revenus, étant donné que les parents noirs occupent des emplois moins bien rémunérês. Or, ces enfants n'ont généralement qu'un parent, et celui-ci est souvent inactif. C'est l'une des interprétations qui se dégagent du tableau 7 , où apparaissent les taux de pauvreté des enfants en fonction de la composition familiale et du travail des parents. Près de $60 \%$ des enfants vivant en famille biparentale sont pauvres si aucun des parents ne travaille; $54 \%$ et $75 \%$ respectivement des enfants de pères ou de mères seuls qui ne travaillent pas sont dans le même cas.

Dans les familles dirigées par un couple marié, on trouve les taux de pauvreté les plus faibles quand les deux parents travaillent à plein temps $(2 \%)$ ou quand le père travaille à plein temps et la mère à temps partiel ( $5 \%$ ). Même lorsque seul le père travaille à plein temps (et que la mère ne fait pas partie de la main-d'œuvre), le taux de pauvreté se situe à $10 \%$, en deçà du taux des enfants vivant en famille monoparentale avec leur père qui travaille à plein temps (13\%), et très en deçà du taux des enfants vivant en famille monoparentale avec leur mère qui travaille à plein temps (19\%). On ne peut qu'en conclure que les ruptures d'union (ainsi que les naissances horsmariage) tendent à frapper les personnes les plus dépourvues de sécurité économique, soit celles qui ont un emploi à plein temps mal payé.

Le tableau 7 permet aussi de distinguer entre les enfants selon leur origine raciale et ethnique. On constate que la pauvreté diminuerait beaucoup chez les enfants américains s'ils vivaient tous dans une famille biparentale où les deux parents travaillent à plein temps. Toutefois, on sait que les minorités ont toujours été moins favorisées au chapitre de la sécurité d'emploi et des taux de salaire et qu'elles ont en outre des familles plus nombreuses, de sorte que même quand elles présentent la même composition familiale et les mêmes modes d'inscription dans l'univers du travail que les blancs, leur revenu est souvent insuffisant. Par exemple, le taux de pauvreté des enfants vivant dans une famille biparentale traditionnelle (où le père fait vivre tout le monde) est de $7 \%$ pour les blancs, de $21 \%$ pour les noirs et de $23 \%$ pour les latino-américains et les amérindiens. Même quand les familles des minorités comptent deux parents qui travaillent, leurs enfants ne peuvent se soustraire à cette condition de défavorisés qui leur échoit en 


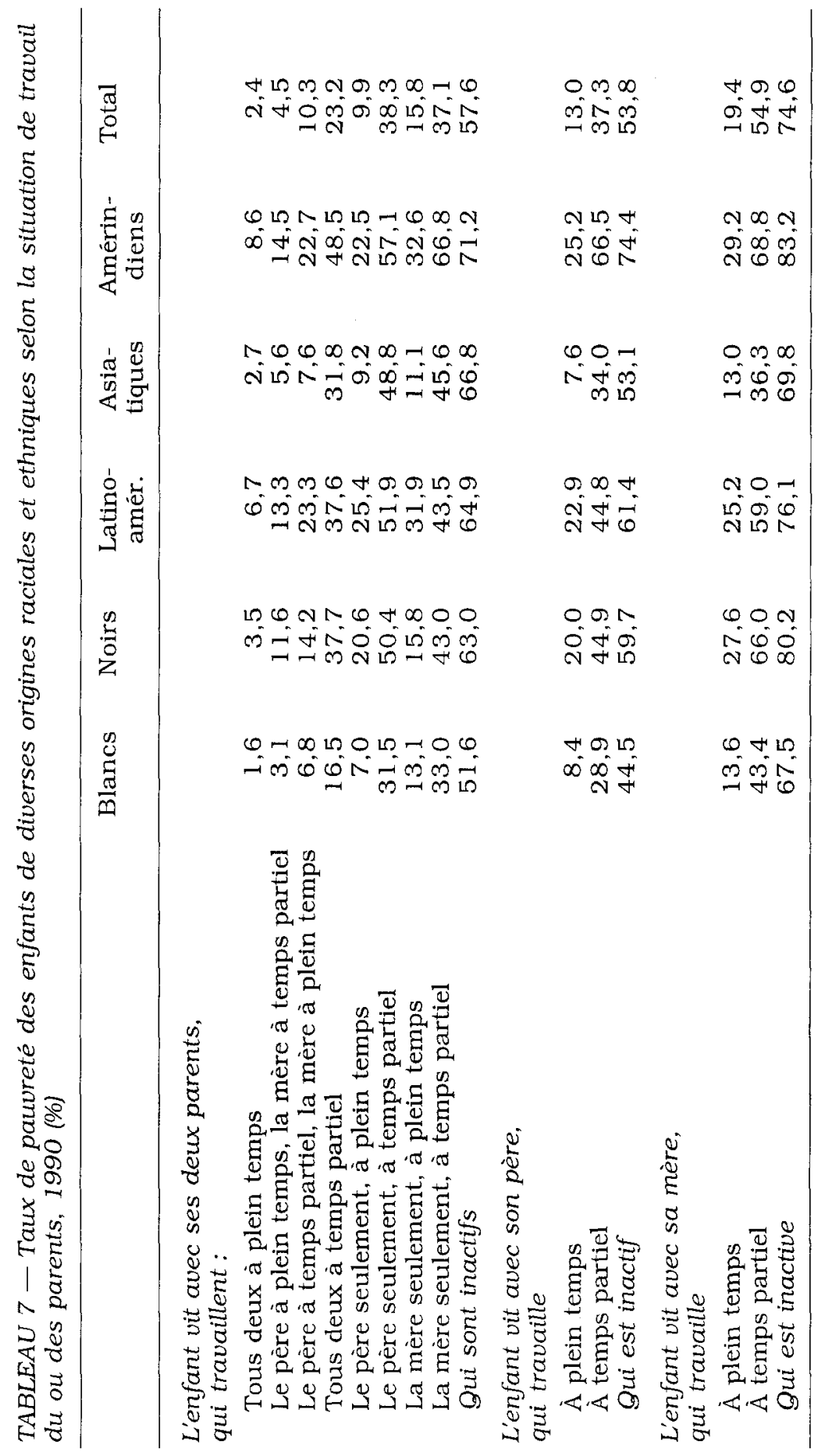


TABLEAU 8 - Taux de pauvreté des enfants de 0 an à 17 ans vivant avec leurs deux parents, leur père seulement et leur mère seulement, normalisés par référence aux modèles de travail des blancs, selon la race ou l'origine ethnique, 1990

\begin{tabular}{|c|c|c|}
\hline & $\begin{array}{c}\text { Taux de pauvreté } \\
\text { normalisé }(\%)\end{array}$ & $\begin{array}{l}\text { Taux normalisé/ } \\
\text { Taux observé }\end{array}$ \\
\hline & \multicolumn{2}{|c|}{ DEUX PARENTS } \\
\hline Noirs & 14,7 & 1,00 \\
\hline Latino-américains & 18,2 & 0,83 \\
\hline Asiatiques & 9,1 & 0,72 \\
\hline \multirow[t]{2}{*}{ Amérindiens } & 18,8 & 0,75 \\
\hline & \multicolumn{2}{|c|}{ PERE } \\
\hline Noirs & 27,7 & 0,82 \\
\hline Latino-américains & 30,1 & 0,91 \\
\hline Asiatiques & 16,1 & 0,81 \\
\hline \multirow[t]{2}{*}{ Amérindiens } & 36,3 & 0,74 \\
\hline & \multicolumn{2}{|c|}{ MÈRE } \\
\hline Noirs & 49,9 & 0,87 \\
\hline Latino-américains & 46,0 & 0,83 \\
\hline Asiatiques & 33,1 & 0,85 \\
\hline Amérindiens & 52,0 & 0,86 \\
\hline
\end{tabular}

héritage. Moins de $2 \%$ des enfants blancs vivant dans de telles familles souffrent de la pauvreté comparativement à près de $4 \%$ des enfants noirs, $7 \%$ des enfants latino-américains et $9 \%$ des enfants amérindiens.

Les données du tableau 7 attestent également que les disparités raciales inscrites dans le travail existent tout aussi bien chez les enfants des familles monoparentales, masculines ou féminines. Les taux de pauvreté normalisés calculés en fonction de la situation de travail (tableau 8) le confirment avec éclat. Ces taux sont établis pour les enfants de chaque minorité raciale ou ethnique, sous l'hypothèse que l'expérience de travail des parents de ces groupes est semblable à celle des parents d'enfants blancs. Le tableau 8 donne aussi le ratio "taux de pauvreté normalisé sur taux de pauvreté observé". Les ratios sont inférieurs à 1,0 si les modèles de travail des parents donnent lieu à une pauvreté notablement plus importante au sein d'un groupe minoritaire.

Ces données montrent que les diffërences entre les parents au chapitre du travail rémunéré ne jouent pas un bien grand rôle dans les variations interraciales ou interethniques de la 
pauvreté chez les enfants. Les taux normalisés des enfants vivant en famille biparentale sont à peine plus bas que leurs taux observés, mais ce faible écart tient en partie au fait que les variations selon la race et l'ethnie dans les modèles de travail des parents sont faibles pour les familles biparentales. D'ailleurs, chez les enfants noirs vivant en famille biparentale, le taux normalisé et le taux observé sont égaux. Ce résultat signifie que les taux de pauvreté de ces enfants noirs, héritiers d'une inégalité raciale qui se perpétue, resteraient 2,4 fois supérieurs à ceux des enfants blancs habitant aussi avec deux parents $(14,7 \%$ contre $6,2 \%)$, même si les parents des uns et des autres participaient de la même manière au travail rémunéré. Chez les enfants latino-américains, le taux de pauvreté passerait de $21,8 \%$ à 18,2 \% dans un tel cas. Les modèles de travail expliquent environ $25 \%$ de la difference entre blancs et latino-américains au chapitre de la pauvreté chez les enfants. Dans le cas des enfants amérindiens, les modèles de travail des parents expliquent environ le tiers des différences interraciales de pauvreté.

Les différences sont plus marquées pour les familles monoparentales. Les enfants noirs vivant avec leur mère verraient leur taux de pauvreté de quelque $57 \%$ passer à $50 \%$ si les mères de ce groupe avaient les mêmes modèles de travail que les mères d'enfants blancs. C'est une diminution de $11 \%$. Cela signifie aussi que les différences entre blancs et noirs dans le travail des mères expliquent près du tiers de l'écart de pauvreté entre enfants blancs et enfants noirs vivant en famille monoparentale avec leur mère.

Dans un sens, les différences de composition familiale et de participation des parents au travail rémunéré comptent parmi les facteurs importants qui expliquent les niveaux supérieurs de pauvreté des enfants des minorités raciales et ethniques. La pauvreté des enfants diminuerait de façon sensible chez les noirs et chez les blancs si tous les enfants vivaient dans une famille biparentale où les deux parents travaillent. Certes, ni une modification de la composition familiale ni une transformation des modèles de travail ne suffiraient à éliminer la pauvreté, mais une évolution positive de ces deux facteurs ferait beaucoup pour réduire la pauvreté dans les groupes de toutes les origines, blancs compris.

D'un autre côté, même si l'on parvenait à atteindre un objectif politiquement plus réaliste (c'est-à-dire à rapprocher les modèles familiaux et les modèles de travail des minorités de 
TABLEAU 9 - Taux de pauvreté des enfants de 0 an à 17 ans, normalisés par référence aux modèles familiaux et aux modèles de travail des blancs, selon la race ou l'origine ethnique, 1990

\begin{tabular}{lcc}
\hline & $\begin{array}{c}\text { Taux de } \\
\text { pauvreté } \\
\text { normalisés } \\
(\%)\end{array}$ & $\begin{array}{c}\text { Taux norma- } \\
\text { lisé/Taux } \\
\text { observé }\end{array}$ \\
\hline Noirs & 20,0 & 0,51 \\
Latino-américains & 22,4 & 0,72 \\
Asiatiques & 12,6 & 0,75 \\
Amérindiens & 23,9 & 0,62 \\
\hline
\end{tabular}

ceux des blancs), des différences substantielles entre les races ou ethnies subsisteraient. Le tableau 9 présente les taux de pauvreté des enfants des minorités, normalisés par rapport aux modèles familiaux et aux modèles de travail des parents des enfants blancs. Si les enfants noirs vivaient dans les mêmes types de familles (biparentales, etc.) que les enfants blancs et avec des parents ayant la même participation au travail que ceux des enfants blancs, leurs taux de pauvreté demeureraient quand même deux fois supérieurs à ceux des blancs $(20,0 \%$ contre $10,5 \%$ ). Dans le même cas, les taux de pauvreté des enfants latino-américains resteraient quant à eux à plus du double de ceux des blancs. Dans certains groupes (chez les noirs par exemple), les taux normalisés équivalent à la moitié environ des taux observés. De toute évidence, la composition familiale et la participation au travail font partie des facteurs qui expliquent les taux de pauvreté élevés de ces enfants, mais d'autres facteurs sont à mettre en cause tout aussi bien.

\section{COMMENTAIRES ET CONCLUSION}

Nous avons voulu mettre à profit les données nouvellement publiées du PUMS du Recensement américain de 1990 pour évaluer les différences de situation économique entre enfants de diverses races et ethnies. Jusqu'à quel point les inégalités raciales sont-elles le produit des différences que l'on constate entre ces groupes au point de vue de la composition des familles et des modes de participation des parents au travail rémunéré ? Cette question est d'autant plus importante que l'on est encore loin d'avoir conclu le débat sur les meilleures politiques à mettre en œuvre pour combattre la pauvreté de la catégorie de 
population la plus vulnérable du pays : les enfants (Haveman et Wolfe, 1993; Bianchi, 1993).

Nos résultats nous amènent à deux conclusions. Premièrement, il est manifeste que les différences de composition familiale entre les groupes sapent les efforts consentis pour éliminer chez les enfants américains les inégalités économiques liées à l'origine raciale et ethnique. "L'échec de la famille" coûte démesurément cher aux enfants noirs surtout, du moins si l'on en juge par les pourcentages de pauvres qu'ils comptent dans leurs rangs : leurs taux de pauvreté diminueraient de $45 \%$ si, dans les mêmes proportions que les enfants blancs, ils vivaient dans des familles dirigées par un couple marié. Deuxièmement, on constate que les différences interraciales dans les modes de participation des parents au travail rémunéré contribuent à expliquer les fortes disparités économiques entre les enfants des divers groupes, mais n'en rendent pas entièrement compte. Dans le cas des familles dirigées par un couple marié, notamment, les différences de participation au travail sont faibles mais les écarts de pauvreté entre enfants souvent très grands. $\mathrm{Si}$ on compare les enfants blancs et les enfants noirs qui vivent dans ce type de famille, on s'aperçoit que les noirs ont des taux de pauvreté à peu près deux fois plus élevés que ceux des blancs, même s'il y a plus de chances que leurs deux parents travaillent.

Ces résultats signifient beaucoup sur le plan des politiques. Une conclusion surtout s'impose avec évidence : les efforts pour "renforcer la famille", en particulier dans le cas des enfants des minorités défavorisées de génération en génération, n'élimineront pas l'inégalité raciale. Nonobstant les types de familles, ces enfants ont des taux de pauvreté plus élevés que les enfants blancs. On est tenté aujourd'hui de ramener le problème de la pauvreté à une "affaire de famille". Notre analyse montre clairement que ce diagnostic n'est pas juste. Néanmoins, les gouvernements ne devraient pas s'abstenir, dans leurs politiques, de promouvoir la famille biparentale en tant que milieu idéal pour mettre au monde et élever des enfants : les enfants de tous les groupes raciaux et ethniques ont des taux de pauvreté beaucoup plus bas s'ils vivent avec deux parents.

Il ne fait pas de doute non plus, selon nos résultats, que les politiques incitant les parents pauvres au travail, même si elles contribuent à la diminution des taux de pauvreté chez les enfants, n'élimineront pas une inégalité raciale persistante. Lichter et Eggebeen (1994), par exemple, ont montré qu'un 
accroissement de $50 \%$ du taux d'emploi des femmes dans les familles pauvres dirigées par un couple marié réduirait d'environ $30 \%$ les taux de pauvreté chez les enfants. Le même accroissement de $50 \%$ du taux d'emploi des mères pauvres chefs de famille monoparentale abaisserait de $26 \%$ le taux de pauvreté des enfants de ce type de famille. C'est dire que la diminution de la pauvreté des enfants serait modeste comparativement à l'augmentation du travail des mères. La raison en est que souvent les gains ainsi obtenus par le travail ne suffisent pas à faire passer familles et enfants au dessus du seuil de pauvreté. La pauvreté des enfants ne diminuera pas de façon significative sans la mise en ouvre de politiques qui combattront "la pauvreté dans le travail". Durant les années 1980, la proportion de travailleurs pauvres a augmenté rapidement, surtout au sein des plus jeunes populations en âge de procreeer (Lichter, Johnston et McLaughlin, 1994).

Heureusement, des efforts sont faits pour rendre le travail plus "rentable". Ainsi, le législateur a élargi la portée du Earned Income Credit ${ }^{5}$ pour faire en sorte que les familles qui comprennent une personne travaillant à plein temps ne soient plus pauvres selon les seuils officiels. La hausse du salaire minimum pourrait également profiter aux enfants pauvres (Bianchi, 1993). Les stratégies de mise en valeur du "capital humain", qui permettent aux mères pauvres de gagner davantage quand elles vont sur le marché du travail, sont une autre possibilité (Corbett, 1993), mais les programmes de formation, tel le Comprehensive Employment and Training Act (CETA) ${ }^{6}$, n'ont eu jusqu'ici qu'un succès mitigé (Haveman et Wolfe, 1993; Garfinkel et McLanahan, 1986). En dernière analyse, il importe de situer le rôle du travail des parents dans une juste perspective. Les incitatifs au travail visant les parents pauvres peuvent améliorer le bien-être économique de certains enfants et sont sans doute souhaitables à d'autres égards, ne serait-ce qu'en valorisant le travail en tant que rôle propre de l'adulte. Mais le travail ne peut à lui seul éliminer ni la pauvreté ni la perpétuation des inégalités raciales chez les enfants américains.

5 Crédit d'impôt lié au revenu de travail (NDLT).

6 Loi sur l'emploi et la formation (NDLT). 


\section{RÉFÉRENCES BIBLIOGRAPHIUES}

BLANCHI, S. M., 1993. "Children of Poverty: Why are They Poor?", dans Judith CHAFEL, éd. Child Poverty and Public Policy. Washington, D. C., Urban Institute, 351 pages.

BIANCHI, S. M., et E. MCARTHUR, 1991. Family Disruption and Economic Hardship: The Short-Run Picture for Children. U. S. Bureau of the Census, Current Population Reports, Series P-70, No. 23. Washington, D. C., U. S. Government Printing Office, 32 pages.

BUMPASS, L., 1984. "Children and Marital Disruption: A Replication and Update", Demography, 21, $1: 71-82$.

CHERLIN, A., 1981. Marriage, Divorce, and Remarriage. Cambridge (MA), Harvard University Press, 142 pages.

CHILDREN'S DEFENSE FUND, 1991. The State of American Children 1991. Washington, D. C., Children's Defense Fund, 170 pages.

CORBETT, T., 1993. "Child Poverty and Welfare Reform: Progress or Paralysis?", FOCUS, 15, $1: 1-17$.

DANZIGER, S., et S. DANZIGER, 1993. "Child Poverty and Public Policy: Toward a Comprehensive Antipoverty Agenda", Daedalus, $122,1: 57-84$.

DUNCAN, G. J., 1991. "The Economic Environment of Childhood", dans A. C. HUSTON, éd. Children in Poverty. Cambridge, Cambridge University Press : 23-50.

DUNCAN, G. J., et W. L. RODGERS, 1991. "Has Children's Poverty Become More Persistent?", American Sociological Review, 56, 4 : 538-550.

EASTERLIN, R. A., 1987. "The New Age Structure of Poverty in America: Permanent or Transient?", Population and Development Review, 13, 2 : 195-207.

EGGEBEEN, D. J., et D. T. LICHTER, 1991. "Race, Family Structure, and Changing Poverty Among American Children", American Sociological Review, 56, $6: 801-817$.

FUCHS, V., et D. REKLIS, 1992. "American's Children: Economic Perspectives and Policy Options", Science, 255, 1 : 41-46.

GALSTON, W. A., 1993. "Causes of Declining Well-Being Among U.S. Children", Aspen Institute Quarterly, 5, 1 : 52-77.

GARFINKEL, I., et S. S. McLANAHAN, 1986. Single Mothers and Their Children. Washington, D. C., The Urban Institute Press, 198 pages.

HAVEMAN, R., et B. WOLFE, 1993. "Children's Prospects and Children's Policy", Journal of Economic Perspectives, 7, 2 : 153174.

HERNANDEZ, D. J., 1993. America's Children: Resources From Family, Government and the Economy. New York (N. Y.), Russell Sage Foundation, 482 pages. 
JENSEN, L., D. J. EGGEBEEN et D. T. LICHTER, 1993. "Child Poverty and the Ameliorative Effects of Public Assistance», Social Science Quarterly, 74, $3: 542-559$.

LANDALE, N. S., et S. HUAUN, 1993. "The Family Life Course of Puerto Rican Children", Journal of Marriage and the Family, 54, 6: 912-924.

LICHTER, D. T., et D. J. EGGEBEEN, 1993. «Rich Kids, Poor Kids: Changing Income Inequality Among American Children", Social Forces, $71,3: 761-780$.

LICHTER, D. T., et D. J. EGGEBEEN, 1994. "The Effect of Parental Employment on Child Poverty", Journal of Marriage and the Family, 56, $4:$ 633-645.

LICHTER, D. T., G. M. JOHNSTON et D. K. MCLAUGHLIN, 1994. "Changing Linkages Between Work and Poverty in Rural America", Rural Sociology, 59, $3: 395-415$.

LICHTER, D. T., et N. S. LANDALE, 1995. "Parental Work, Family Structure, and Poverty Among Latino Children", Journal of Marriage and the Family, 57, $2: 346-354$.

MACUNOVICH, D. J, et R. A. EASTERLIN, 1990. "How Parents Have Coped: The Effect of Life Cycle Demographic Decisions on the Economic Status of Pre-School Age Children, 1964-87", Population and Development Review, 16, 2: 301-325.

MCLANAHAN, S. S., 1985. "Family Structure and the Reproduction of Poverty", American Journal of Sociology, 90, $4:$ 873-903.

MEAD, L. M., 1993. The New Politics of Poverty: The Nonworking Poor in America. New York (N. Y.), Basic Books, 356 pages.

MURDOCK, S. H., et D. R. ELLIS, 1991. Applied Demography. Boulder (CO), Westview Press, 299 pages.

NATIONAL COMMISSION ON CHILDREN, 1991. Beyond Rhetoric: A New American Agenda for Children and Families. Washington, D. C., U. S. Government Printing Office, 519 pages.

PRESTON, S. H., 1984. "Children and the Elderly: Divergent Paths for America's Dependents», Demography, 21, 4 : 435-457.

U. S. BUREAU OF THE CENSUS, 1993. Poverty in the United States: 1992. Current Population Reports, Series P60-185. Washington, D. C., U. S. Government Printing Office, 174 pages. 


\section{RÉSUMÉ - SUMMARY - RESUMEN}

LICHTER DANiel T. - ENVIRONNEMENT FAMILIAL ET BIEN-ẼTRE ÉCONOMIQUE DES ENFANTS AMERICAINS

À partir de nouvelles données américaines tirées de l'échantillon à $1 \%$ du Public Use Microdata Sample du Recensement décennal de 1990, l'auteur cherche à mesurer à quel point les variations interraciales du bien-être économique chez les enfants sont liées à des différences entre les groupes de diverses ethnies ou races au point de vue de la composition familiale et (ou) de la participation des parents au travail rémunéré. Il apparait que les différences interraciales de composition familiale rendent inopérants les efforts entrepris pour éliminer les écarts de richesse entre enfants américains de diverses origines. Ainsi, l'importance de la proportion d'enfants noirs vivant en famille monoparentale avec leur mère explique $60 \%$ de l'écart qui sépare le taux de pauvreté des enfants noirs de celui des enfants blancs. De même, les écarts de pauvreté chez les enfants entre les groupes s'expliquent en partie (mais non complètement) par la variation interraciale des modèles de participation au travail des parents. Parmi les enfants vivant en famille biparentale, les taux de pauvreté sont environ deux fois plus élevés chez les enfants noirs que chez les enfants blancs, même si les premiers ont plus de chances d'avoir deux parents qui travaillent. On peut en conclure que les inégalités raciales ne seront pas éliminées par les politiques strictement conçues pour "renforcer la famille" ou inciter les mères au travail (sans égard au niveau des salaires), surtout dans le cas des enfants des minorités défavorisées depuis plusieurs générations.

\section{LICHTER Daniel T. - THE LIVING ARRANGEMENTS AND ECONOMIC WELL- BEING OF AMERICAN CHILDREN}

The objective of this paper is to evaluate the extent to which racial variation in children's economic well-being resides in divergent parental work patterns and/or family living arrangements. This is accomplished using recently-released data from the 1 percent sample of the Public Use Microdata Sample of the 1990 decennial census. The results indicate that racial differences in family structure undermine efforts to eliminate racial inequality among American children. Among blacks, for example, the high proportions of children living in female-headed families account for 60 percent of the difference from white children in poverty rates. Similarly, racial differences in parental work patterns contribute to (but cannot explain completely) racial variation in child poverty. Among black children in married-couple families, poverty rates are roughly twice those of their white counterparts, even though black children are more likely to have both parents working. The implication is that policies narrowly designed to "strengthen the family" or promote maternal employment (without regard to wage levels), especially among historically disadvantaged minority children, will not eliminate racial inequality. 
LICHTER DANiel T. - MEDIO FAMILIAR Y BIENESTAR ECONÓMICO DE LOS NINTOS AMERICANOS

El autor busca aqui evaluar, por medio de los recientes datos americanos del muestreo a 1 por ciento del Public Use Microdate Sample del Censo decenal de 1990, hasta qué punto están relacionadas las variaciones interraciales del bienestar económico de los niños con las diferencias entre distintos grupos raciales según la composición familiar y/o la participación de los padres a la actividad remunerada. Los resultados indican que la diferencias raciales en la estructura familiar vuelven inoperantes los esfuerzos por eliminar las desigualdades entre niños americanos de distintas raices. Por ejemplo, la gran proporción de niños de raza negra que viven en familia monoparental únicamente con la madre explica $60 \%$ de la diferencia en las tasas de pobreza respecto a los niños blancos. Asimismo, las diferencias raciales en el tipo de participación de los padres al empleo remunerado explican en parte (aunque no del todo) las variaciones de pobreza entre los niños. Las tasas de pobreza son dos veces más elevadas en el caso de niños de raza negra que viven en familias con ambos padres (biparentales) que en el caso de niños de raza blanca, aún si en el primer caso en más probable que ambos padres trabajen. Puede deducirse que las desigualdades raciales no se verán eliminadas por politicas dirigidas a "reforzar la familia" o a fomentar el trabajo materno (sin preocuparse de los niveles de sueldos), sobre todo en el caso de niños que forman parte de minorias desfavorecidas desde varias generaciones. 